\title{
Failed Compensatory Dendritic Growth as a Pathophysiological Process in Alzheimer's Disease
}

\author{
Dorothy G. Flood and Paul D. Coleman
}

\begin{abstract}
In normal human aging the remaining neurons of two areas of the hippocampal region have been found to compensate for age-related neuronal loss by proliferating new dendrites. In Alzheimer's disease (AD) the layer ll pyramidal neurons of the parahippocampal gyrus fail to show this compensatory response, in spite of a probable, exaggerated disease-related loss of neurons. In AD the dentate gyrus granule cells of the hippocampus also show a reduced amount of the compensatory response. This failure of the AD brain to show the normal compensatory plastic response, seen in normal aging as dendritic growth, may be viewed as one of the pathophysiological processes of the disease.
\end{abstract}

\begin{abstract}
RÉSUMÉ: Défaillance de la croissance compensatoire des dendrites comme processus pathophysiologique dans la maladie d'Alzheimer. Au cours du vieillissement normal, on a constaté que les neurones restants au niveau de deux aires de la région de l'hippocampe compensent pour la perte neuronale due au vieillissement par la prolifération de nouveaux dendrites. Dans la maladie d'Alzheimer (MA) les neurones pyramidaux de la couche II du gyrus du parahippocampe ne manifestent pas cette réponse compensatoire, en dépit d'une perte de neurones probablement exagérée, en rapport avec la maladie. Dans la $\mathrm{MA}$, les cellules à granulations du gyrus dentatus de l'hippocampe manifestent également une réponse compensatoire atténuée. La réponse plastique compensatoire normale, c'est-àdire la croissance dendritique telle qu'on la voit dans le vieillissement normal, fait défaut dans le cerveau atteint de MA. Cette absence de réponse peut être considérée comme un des processus pathophysiologiques de la maladie.
\end{abstract}

Can. J. Neurol. Sci. 1986; 13:475-479

In the human central nervous system normal age-related loss of neurons has been reported in a variety of regions, including all areas of the cerebral cortex ${ }^{1.2 .3,4.5}$ and the hippocampus $^{5,6,7}$ that have been examined to date. In the hippocampal region, areas that lose neurons include Ammon's horn, ${ }^{5,6.7}$ dentate gyrus, ${ }^{7}$ and subiculum. ${ }^{2}$ This age-related neuronal loss causes the deafferentation of the neurons that are the targets of these dying neurons. Additionally, the freeing of afferents by loss of the postsynaptic target neurons has been hypothesized to cause the elongation of the dendritic trees of neighboring, surviving neurons in normal aging, as remaining dendrites expand to engage unoccupied afferents. ${ }^{89}$ This ability of aging neurons to compensate for neuronal loss by dendritic growth and modification of connections, is thought to represent a continuation of the process of neuronal plasticity begun by the nervous system during early development. ${ }^{10}$ The phenomenon of dendritic plasticity in aged subjects has been demonstrated in the human, ${ }^{11,12}$ in a subhuman primate, ${ }^{13,14}$ and in the rat. ${ }^{8}$ In aging human, dendritic growth was first found in layer II pyramidal neurons of the parahippocampal gyrus between middle age (mean age $=51.2$ years) and old age (mean age $=79.6$

\section{years). ${ }^{11,12,15}$}

Buell and Coleman ${ }^{11.12 .15}$ have also studied dendrites of the same cell type in patients with Alzheimer's disease (AD). Dendritic trees of layer II pyramidal neurons of parahippocampal gyrus were found to be significantly smaller in the AD group (mean age $=76.0$ years) than in the normal old-aged group (mean age $=79.6$ years). The AD and middle-aged (mean age $=51.2$ years) groups were similar in most measures of dendritic extent; but small differences between these two groups were statistically significant for some measures. When there were differences between the AD and the middle-aged groups, the dendritic trees in the $\mathrm{AD}$ group were smaller than those in the middle-aged group. Thus, in spite of the possibility that more neurons are lost in the parahippocampal gyrus in AD than in normal aging, there was a failure to show any compensatory plastic dendritic growth.

In examining an additional cell type in the hippocampal region in largely the same case material as used for the study of layer II pyramids of parahippocampal gyrus, ${ }^{11.12 .15}$ we have recently found some evidence for dendritic plasticity in the AD brain. In granule cells of the dentate gyrus, increased numbers of den- 
dritic segments and reduced segment lengths have been found, suggesting a reduced or aberrant form of plasticity may still remain in some brain regions in $\mathrm{AD} .^{16}$

\section{METHODS}

Twenty-two human cases were obtained at autopsy and divided into four groups, three normal groups of varying ages and one group with Alzheimer's disease. A normal middle-aged group (MA, $N=7$ ) averaged 52 years of age, with a range from 43 to 60 years old. A normal old-aged group (OA, $N=5$ ) averaged 73 years of age, with a range from 68 to 79 years old. A normal very old-aged group (VOA, $N=5$ ) averaged 90 years of age, with a range from 86 to 95 years old. A group with Alzheimer's disease ( $A D, N=5$ ) averaged 76 years of age, with a range from 70 to 81 years old. Normal cases had a clinical history with no evidence of neurological or psychiatric symptoms, and were alert, well oriented, and capable of functioning relatively independently shortly before death. AD cases had the diagnosis of a severe, progressive dementia, but were free of other severe neurological and psychiatric abnormalities. The AD cases also had abundant Alzheimer's changes (senile plaques, neurofibrillary tangles, or granulovacuolar degeneration) in the hippocampal region. Some of the normal cases had mild amounts of Alzheimer's changes in the hippocampal region, with the older cases having more changes. All cases were free of other neuropathological findings, such as stroke, tumour, trauma, etc. Additional information on the cases with regard to age, sex, postmortem delay, Alzheimer's changes, cause of death, and clinical history is available elsewhere. ${ }^{15.16}$

Tissue from the right hippocampus, taken just posterior to the uncus, was stained by the Van der Loos ${ }^{17}$ modification of the Golgi Cox method. Frontal sections were cut at $200 \mu \mathrm{m}$ and processed as previously described. ${ }^{12,17}$ Tissue from the left hippocampus was fixed in formalin and stained with hematoxylin and eosin for routine pathological survey, or by the Bodian method $^{18}$ for survey of Alzheimer's changes.

Golgi-impregnated sections from each case were coded; and fifteen neurons per case were chosen randomly for analysis from all portions of the ' $C$ ' of the dentate gyrus. The presence of cut dendritic segments on a neuron was not a criterion for its exclusion from the sample. Cut segments accounted for less than $10 \%$ of the entire population of terminal segments. The sample of neurons for all subjects included those which were normal and robust in appearance, as well as those which were grossly atrophic. The appearance of a neuron was not a criterion for its selection or elimination from the sample. Along with a $100 \mu \mathrm{m}$ calibration line, the outline of the cell body and the entire apical dendritic tree system were drawn with a camera lucida at 200 or $400 \times$ and entered into an Apple II Plus microcomputer by tracing on a graphics tablet. Basal dendritic trees, those which course towards or enter the hilus, ${ }^{19}$ were not drawn or analyzed. Total dendritic length, number of segments, and average segment length (total dendritic length/number of segments) were quantified by computer for each cell. Data from the 15 cells per case were averaged; and statistical tests were performed with $\mathrm{N}$ equal to the number of subjects.

\section{RESULTS}

Figure 1 shows the means and standard errors of the means for the granule cell apical dendritic tree system for each group

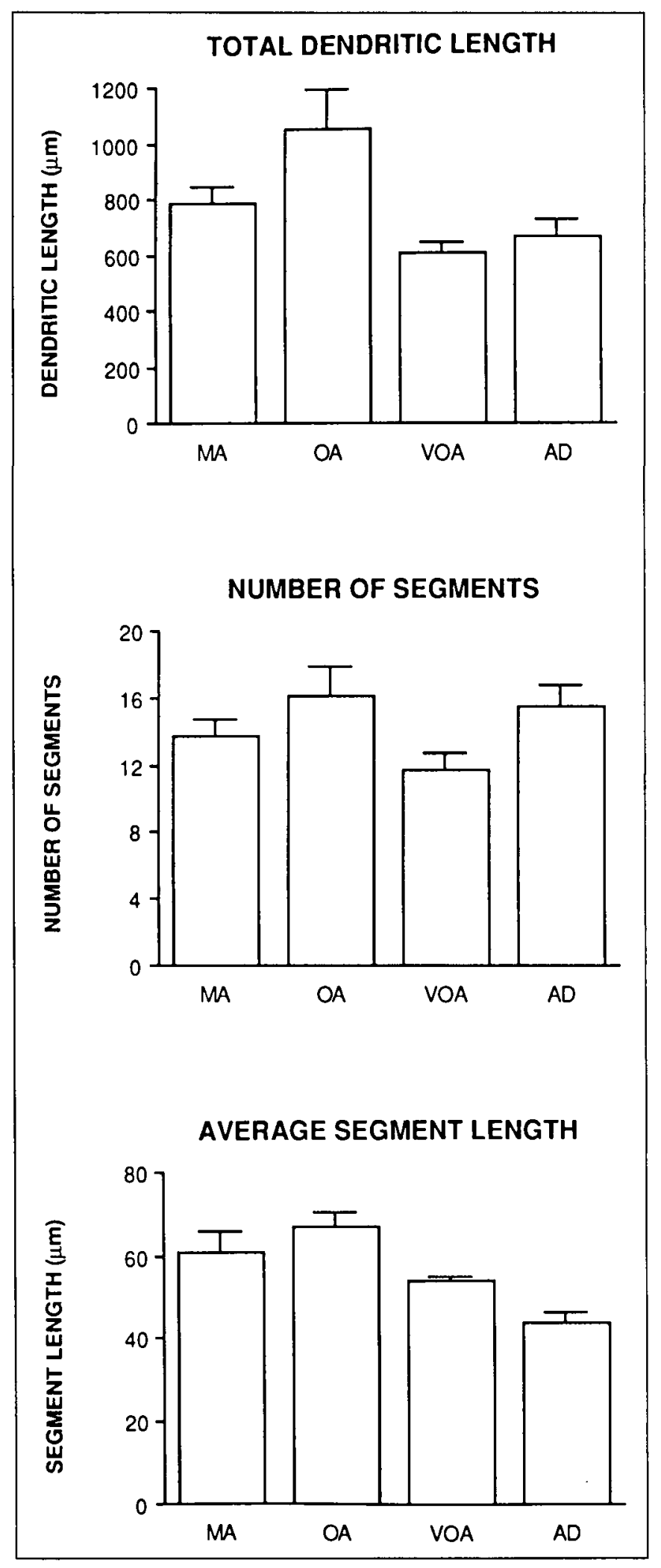

Figure 1 - Total dendritic length, number of segments, and average segment length for the apical dendritic trees of dentate gyrus granule cells. MA - middle-aged, OA - old-aged. VOA - very old-aged, and $A D$ Alzheimer's disease groups. Error bars represent standard error of the mean. Results of the ANOVA's with 3 and 18 degrees of freedom are: totaldendriticlength $F=6.52, p<0.005$; number of segments, $\mathrm{F}=2.81, \mathrm{p}>0.10 ;$ and average segment length. $F=6.40, p<0.005$. 
for the measures of total dendritic length, number of segments, and average segment length. Total dendritic length increased from middle age to old age, and then decreased from old age to very old age. The very old-aged subjects had $23 \%$ less total dendritic extent than the middle-aged subjects. The AD subjects had $16 \%$ less total dendritic length than the middle-aged subjects, $37 \%$ less than the old-aged subjects, the comparably aged subjects, and $10 \%$ more than the very old-aged subjects. These group differences in total dendritic extent were found to be highly statistically significant using a parametric analysis of variance (ANOVA) (Figure 1). Post hoc comparisons with the Newman-Keuls test showed that total dendritic length for the old-aged group was significantly different from that for the middle-aged, very old-aged, and AD groups $(p<0.05)$. The latter three groups did not vary from one another for the measure of total dendritic length.

The total number of segments for the granule cell apical dendritic trees did not vary significantly among the groups (Figure 1). The higher numbers of segments were found in the old-aged and AD groups, and the lowest number in the very old-aged group. Average segment length (total dendritic length/ number of segments) was found to be significantly different (Figure 1). The AD group was significantly $(p<0.05)$ lower in average segment length than the middle-and old-aged groups, as shown with Newman-Keuls post hoc test.

These data suggest that the normally aging granule cells of the human dentate gyrus show significant changes in total dendritic length that are the result of concordant nonsignificant changes in both the numbers and lengths of dendritic segments. In comparing the granule cell apical dendritic trees of the $\mathrm{AD}$ group with those of the normal comparably aged (OA) and younger (MA) groups, there was a significant reduction in the $A D$ group in average segment length with no loss in the numbers of segments.

\section{Discussion}

Both proliferative and regressive factors influence dendrites in the aging brain. ${ }^{9}$ Dendritic proliferation may be induced as a result of the death of neighbor neurons, while dendritic regression may be induced by the loss of afferent supply. ${ }^{9}$ The observed outcome for dendritic extent in a given cell type, either net regression, net stability, or net growth of dendrites, may depend on the balance between these influences in the local environment of the neurons. For layer II pyramidal neurons of human parahippocampal gyrus, dendritic growth was found into old age. Beyond the eighth decade of life, the growth was followed by either net dendritic stability or further growth in very old age. ${ }^{15}$ Because there were only two very old subjects, it is impossible to distinguish between net dendritic stability and growth at the present time for layer II pyramids of the parahippocampal gyrus. For granule cells of the human dentate gyrus, normal age-related dendritic growth between middle age and old age is followed by dendritic regression between old age and very old age. Reports on age-related dendritic changes in animal studies also show similar regional variability. In rat, for instance, studies on a variety of cell types have found dendritic growth, ${ }^{20,21}$ regression, ${ }^{22,23,24,25}$ growth followed by regression, ${ }^{8}$ or net stability. ${ }^{26.27}$ These regional specificities in dendritic changes provide further support for the notion that agerelated changes in dendritic extent may be due to factors in the local environment, such as the balance between death of neighboring neurons and changes in the afferent supply to the neurons. ${ }^{9}$

In Alzheimer's disease, dendritic extent in dentate gyrus granule cells was significantly less than in the comparably aged normal group (OA group). The reduction in total dendritic length in the AD group when compared with the old-aged group was largely due to a significant reduction in average segment length, and very slightly due to a nonsignificant reduction in numbers of segments. Despite the fact that the AD group did not have significantly less total dendritic extent than the middleaged group, average segment length was significantly reduced in the $\mathrm{AD}$ group compared with the middle-aged group. Because average segment lengths in the $\mathrm{AD}$ group were reduced compared with the middle-aged group, a process of real dendritic regression may have been taking place. The granule cells in the AD group compensated for the reduced average segment length by increasing the numbers of segments, yielding a similar picture for the $\mathrm{AD}$ and middle-aged groups for total dendritic extent. No measures of the granule cell dendrites were significantly different between the AD and very old-aged group. Although total dendritic length for the two groups was quite similar, the AD group had greatly reduced average segment lengths, but larger numbers of segments when compared with the very old-aged group. Because of the dissimilarities in the patterns of dendritic change in $A D$ and in normal aging to a very old age, it is unlikely that $A D$, in this cell population, represents a process of accelerated neuronal aging.

Because there were increased numbers of segments in the $\mathrm{AD}$ group compared with the middle-aged group, we suggest that at some time between middle age and death, the AD cases did show a plastic compensatory response. The compensatory response may have occurred either before the onset of $A D$ or during its course. The compensatory response, however, was not normal, since the AD cases had fewer segments than the comparably aged normal group (OA group). Our present data did not permit the determination of whether the $A D$ cases lost dendritic segments or simply failed to proliferate as many as the old-aged cases. Additionally, the plastic response may have been aberrant since the average segment lengths in the $A D$ cases were significantly reduced when compared with the comparably aged normal group. Despite questions regarding the robustness of the plastic response or its comparability to plasticity in normal aging, the maintenance of some plastic capacity in AD between middle age and the terminal stages of the disease has been found for dentate gyrus granule cells. Further evidence that the dentate gyrus granule cells and their commissural and associational afferents remain plastic in $A D$ has recently been demonstrated. ${ }^{28}$

The plastic alterations seen in the dentate gyrus granule cells in $\mathrm{AD}$ are probably induced by the slow, continual loss of their perforant path input to the outer molecular layer from the entorhinal cortex. ${ }^{28.29}$ The cells of origin of the perforant path, ${ }^{30.31}$ the neurons of layer II of the entrohinal cortex, are well known to accumulate neurofibrillary tangles and to eventually degenerate. ${ }^{29.32}$ Remaining afferents in the inner molecular layer grow to occupy the vacated synaptic territory on the dendrites of the granule cells. ${ }^{28}$ This afferent growth coincides with the dendritic plasticity in the dentate gyrus granule cells that we have observed in the AD cases. 
Dendrites form as much as $95 \%$ of the receptive surface that a neuron offers for contact with other neurons. ${ }^{33}$ Thus, maintenance of the ability for dendritic growth and remodeling in aging is possibly an important factor for the integrity of neuronal connections and of the central nervous system as a whole. In the normal human aging brain, dendritic growth has been reported in two cell types of the hippocampal region. ${ }^{11.12,16,34}$ In $A D$, a lack of dendritic growth has been found in the parahippocampal gyrus layer II pyramidal neurons; ${ }^{11.12}$ and an aberrant form of dendritic growth has been found in the granule cells of the dentate gyrus. ${ }^{16}$ This lack of normal plastic dendritic proliferation in response to neuronal loss in AD may be an important contributing factor in the breakdown of interneuronal connections and in the observed deterioration of cognitive functions. The fact that total dendritic length in dentate gyrus granule cells in $\mathrm{AD}$ is comparable to that seen in the mentally functional very old-aged cases suggests that static dendritic extent is not a determining variable in the behavioral devastation of $\mathrm{AD}$. However, failure of plasticity, as well as other variables related to deficits in neurotransmission, may be more closely related to the reduced behavioral capacity in $\mathrm{AD}$. The work of Geddes et $\mathrm{al}^{28}$ has suggested that the maintenance of plasticity in the dentate gyrus in AD is dependent upon an intact cholinergic innervation from the basal forebrain. Thus, the deficits we observe in plasticity of dendrites in $A D$ in the hippocampal region may be related to the well known deficits in the cholinergic system produced by neuronal loss in the basal forebrain. ${ }^{35.36 .37 .38 .39 .40 .41}$ And, failure of dendritic plasticity in $\mathrm{AD}$ may represent one of the pathophysiological mechanisms leading to the inevitable functional decline of the $A D$ brain since it has been robbed of one of the repair mechanisms available to the normally aging brain.

\section{ACKNOWLEDGEMENT}

This work was supported by Public Health Service grants AG 02680, AG 01121 , and AG 03644.

\section{REFERENCES}

I. Brody H. Organization of the cerebral cortex. III. A study of aging in the human cerebral cortex. J Comp Neurol 1955; 102: 511-556.

2. Shefer VF. Absolute number of neurons and thickness of the cerebral cortex during aging, senile and vascular dementia, and Pick's and Alzheimer's diseases. Neurosci Behav Physiol 1973; 6: 319-324.

3. Henderson G, Tomlinson BE, Gibson PH. Cell counts in human cerebral cortex in normal adults throughout life using an image analysing computer. J Neurol Sci 1980; 46: 113-136.

4. Anderson JM, Hubbard BM, Coghill GR, et al. The effect of advanced old age on the neurone content of the cerebral cortex. Observations with an automatic image analyser point counting method. J. Neurol Sci 1983; 58: 233-244.

5. Mann DMA, Yates PO, Marcyniuk B. Some morphometric observations on the cerebral cortex and hippocampus in presenile Alzheimer's disease, senile dementia of Alzheimer type and Down's syndrome in middle age. J Neurol Sci 1985; 69: 139-159.

6. Ball MJ. Neuronal loss, neurofibrillary tangles and granulovacuolar degeneration in the hippocampus with ageing and dementia. A quantitative study. Acta Neuropathol (Berl) 1977; 37: 111-118.

7. Mouritzen Dam A. The density of neurons in the human hippocampus. Neuropathol Appl Neurobiol 1979; 5: 249-264.

8. Hinds JW, McNelly NA. Aging of the rat olfactory bulb: Growth and atrophy of constituent layers and changes in size and number of mitral cells. J Comp Neurol 1977; 171: 345-368.
9. Coleman PD, Flood DG. Dendritic proliferation in the aging brain as a compensatory repair mechanism. Prog Brain Res 1986; 70: 227-237.

10. Coleman PD, Buell SJ. Regulation of dendritic extent in developing and aging brain. In: Cotman $\mathrm{CW}$, ed. Synaptic Plasticity. New York: The Guilford Press, 1985: 311-333.

11. Buell SJ, Coleman PD. Dendritic growth in the aged human brain and failure of growth in senile dementia. Science 1979; 206: 854-856.

12. Buell SJ, Coleman PD. Quantitative evidence for selective dendritic growth in normal human aging but not in senile dementia. Brain Res 1981; 214: 23-41.

13. Cupp CJ, Uemura E. Age-related changes in prefrontal cortex of Macaca mulatta: Quantitative analysis of dendritic branching patterns. Exp Neurol 1980; 69: 143-163.

14. Uemura $\mathrm{E}$. Age-related changes in the subiculum of Macaca mulatta: Dendritic branching pattern. Exp Neurol 1985; 87: 412-427.

15. Buell SJ, Coleman PD. Individual differences in dendritic growth in human aging and senile dementia. In: Stein D, ed. The Psychobiology of Aging: Problems and Perspectives. Amsterdam: Elsevier, 1980: 283-296.

16. Flood DG, Buell SJ, Horwitz GJ, et al. Dendritic extent in human dentate gyrus granule cells in normal aging and senile dementia. Brain Res 1986 (in press).

17. Van der Loos H. Une combinaison de deux vieilles methodes histologiques pour le systeme nerveux central. Monatsschr Psychiatr Neurol 1956; 132; 330-334.

18. Bodian D. A new method for staining nerve fibers and nerve endings in mounted paraffin sections. Anat Rec 1936; 65: 89-97.

19. Lindsay RD, Scheibel AB. Quantitative anaylsis of dendritic branching pattern of granular cells from human dentate gyrus. Exp Neurol 1976; 52: 295-310.

20. Connor JR, Diamond MC, Johnson RE. Occipital cortical morphology of the rat: Alterations with age and environment. Exp Neurol 1980; 68: 158-170.

21. Connor JR, Beban SE, Hopper PA, et al. A Golgi study of the superficial pyramidal cells in the somatosensory cortex of socially reared old adult rats. Exp Neurol 1982; 76: 35-45.

22. Hinds JW, McNelly NA. Aging in the rat olfactory system: Correlation of changes in the olfactory epithelium and olfactory bulb. J Comp Neurol 1981; 203: 441-453.

23. Flood DG, Coleman PD. Age-related changes in dendritic extent of neurons in supraoptic nucleus of F344 rats. Soc Neurosci Abstr 1983; 9: 930.

24. Rogers J, Zornetzer SF, Bloom FE, et al. Senescent microstructural changes in rat cerebellum. Brain Res 1984; 292: 23-32.

25. Pentney RJ. Quantitative analysis of dendritic networks of Purkinje neurons during aging. Neurobiol Aging 1986 (in press).

26. Pysh JJ, Benson MD. Purkinje cell dendrites in aged rats: A morphometric Golgi analysis. Soc Neurosci Abstr 1980; 6: 281.

27. Coleman PD, Buell SJ. Dendritic extent of layer II pyramids in entorhinal cortex of aging F344 rat. Soc Neurosci Abstr 1983;9: 930.

28. Geddes JW, Monaghan DT, Cotman CW, et al. Plasticity of hippocampal circuitry in Alzheimer's disease. Science 1985; 230: $1179-1181$.

29. Hyman BT, Van Hoesen GW, Damasio AR, et al. Alzheimer's disease: Cell-specific pathology isolates the hippocampal formation. Science 1984; 225: 1168-1170.

30. Lorente de No R. Studies on the structure of the cerebral cortex. II. Continuation of the study of the ammonic system. J Psychol Neurol (Leipzig) 1934; 46: 113-177.

31. Van Hoesen GW, Pandya DN. Some connections of the entorhinal (area 28) and perirhinal (area 35) cortices of the rhesus monkey. III. Efferent connections. Brain Res 1975; 95: $39-59$.

32. Hirano A, Zimmerman HM. Alzheimer's neurofibrillary changes A topographic study. Arch Neurol 1962; 7: 227-242.

33. Schade JP, Baxter CF. Changes during growth in the volume and surface area of cortical neurons in the rabbit. Exp Neurol 1960; 2: $158-178$.

34. Flood DG, Buell SJ, DeFiore $\mathrm{CH}$, et al. Age-related dendritic growth in dentate gyrus of human brain is followed by regression in the 'oldest old'. Brain Res 1985; 345: 366-368. 
35. Whitehouse PJ, Price DL, Struble RG, et al. Alzheimer's disease and senile dementia: Loss of neurons in the basal forebrain. Science 1982; 215: 1237-1239.

36. Candy JM, Perry RH, Perry EK, et al. Pathological changes in the nucleus of Meynert in Alzheimer's and Parkinson's diseases. J Neurol Sci 1983; 54: 277-289.

37. Tagliavini F, Pilleri G. Basal nucleus of Meynert. A neuropathological study in Alzheimer's disease, simple senile dementia, Pick's disease and Huntington's chorea. J Neurol Sci 1983; 62: 243-260.

38. Wilcock GK, Esiri MM, Bowen DM, et al. The nucleus basalis in Alzheimer's disease: Cell counts and cortical biochemistry. Neuropathol Appl Neurobiol 1983; 9: 175-179.
39. Mann DMA, Yates PO, Marcyniuk B. Changes in nerve cells of the nucleus basalis of Meynert in Alzheimer's disease and their relationship to ageing and to the accumulation of lipofuscin pigment. Mech Ageing Dev 1984; 25: 189-204.

40. McGeer PL, McGeer EG, Suzuki J, et al. Aging, Alzheimer's disease, and the cholinergic system of the basal forebrain. Neurology 1984; 34: 741-745.

41. Arendt T, Bigl V, Tennstedt A, et al. Neuronal loss in different parts of the nucleus basalis is related to neuritic plaque formation in cortical target areas in Alzheimer's disease. Neuroscience $1985 ; 14: 1-14$. 